\title{
THE CONJUGACY PROBLEM FOR GRAPH PRODUCTS WITH FINITE CYCLIC EDGE GROUPS
}

\author{
JODY MEYER LOCKHART \\ (Communicated by Warren J. Wong)
}

\begin{abstract}
Finite graph products of groups with solvable conjugacy problem and with finite cyclic edge groups are considered. It is shown that the graph product has solvable conjugacy problem if each of the vertex groups has solvable conjugacy problem.
\end{abstract}

The conjugacy problem for graph products (the fundamental group of a graph of groups as defined by Bass and Serre; see Serre [2]) was studied by Horadam [1]. Horadam showed that finite graph products with finite cyclic edge groups inherit a solvable conjugacy problem from their vertex groups under certain conditions, one of which is that the images of the edge group generators in each vertex group are powers of a common central element. We show that this condition is not necessary and, in fact, that all finite graph products with finite cyclic edge groups inherit a solvable conjugacy problem from their vertex groups.

Let $D$ be a finite connected directed graph with edge set $E$, vertex set $V$, and source and terminus maps $s$ and $t$, respectively. Let $(G, D)$ be a graph of groups; that is, for each $v \in V$, there is a vertex group $G_{v}$ and for each $e \in E$, there is an edge group $G_{e}$. Further, for each $e \in E$, there are partially recursive monomorphisms $A_{e}: G_{e} \rightarrow G_{s e}$ and $B_{e}: G_{e} \rightarrow G_{t e}$. Let $T$ be a maximal tree of $D$ and let $E^{\prime}$ be the edge set of $T$. Then the graph product $G^{*}$ has presentation

$$
\begin{aligned}
G^{*}= & \left\langle G_{v}(v \in V), e(e \in E) ;\right. \\
& \left.e B_{e}(x) e^{-1}=A_{e}(x)\left(x \in G_{e}, e \in E\right), e=1\left(e \in E^{\prime}\right)\right\rangle .
\end{aligned}
$$

Note that $G^{*}$ is a finite sequence of $\mathrm{HNN}$-extensions (one for each $e$ in $E-E^{\prime}$ ) of a tree product

$$
G_{T}^{*}=\left\langle G_{v}(v \in V) ; A_{e}(x)=B_{e}(x)\left(x \in G_{e}, e \in E^{\prime}\right)\right\rangle,
$$

which is itself a finite sequence of free products with amalgamation (one for each $e$ in $E^{\prime}$ ).

We will consider graph products such that for each $e \in E, G_{e}$ is a finite cyclic group $\left\langle k_{e}\right\rangle$.

Received by the editors July 10, 1990 and, in revised form, July 16, 1991.

1991 Mathematics Subject Classification. Primary 20E06, 20F10; Secondary 20 F05.

Research supported by a grant from the Naval Academy Research Council 
Lemma. If $H$ has solvable conjugacy problem and if $a$ and $b$ are elements of $H$ both of finite order $n$, then the $H N N$-extension $G=\left\langle H, t ; t^{-1}\right.$ at $\left.=b\right\rangle$ has solvable conjugacy problem.

Proof. First note that, since $a$ and $b$ are of finite order and since $H$ has solvable word problem, the membership problems for $\langle a\rangle$ in $H$ and for $\langle b\rangle$ in $H$ are solvable. Hence, $G$ has solvable word problem.

Now suppose $U, V \in H$. It is known that $U \sim_{G} V$ if and only if $U \sim_{H} V$ or there is a chain

$$
U \sim_{H} c_{1}^{n_{1}} \sim_{t} c_{2}^{n_{2}} \sim_{H} c_{3}^{n_{3}} \sim_{t} \cdots \sim V,
$$

where $\sim_{H}$ indicates conjugacy in $H, \sim_{t}$ indicates conjugation by $t$ or $t^{-1}$, each $c_{i}$ is $a$ or $b$, and $c_{2 j-1} \neq c_{2 j}$. If $U$ and $V$ are conjugate in $G$, then there exists such a chain with no repetitions. Since $a$ and $b$ have finite order, there are only finitely many chains with no repetition. Each of these finite number of chains can be effectively checked for validity since $H$ has solvable conjugacy problem.

Now suppose that $U$ and $V$ are cyclically $t$-reduced elements of $G-H$. By Collin's Lemma, $U \sim_{G} V$ if and only if there are cyclic permutations $U^{\prime}$ and $V^{\prime}$ of $U$ and $V$, respectively, and an element $c \in\langle a\rangle \cup\langle b\rangle$ such that $c^{-1} U^{\prime} c=V^{\prime}$. Since there are only finitely many possible $U^{\prime}, V^{\prime}$, and $c$ and since $G$ has solvable word problem, this can be effectively checked.

A similar proof shows that if $H$ and $K$ have solvable conjugacy problem and $a \in H$ and $b \in K$ have finite order $n$, then the free product with amalgamation $\langle H, K ; a=b\rangle$ has solvable conjugacy problem. If $D$ is the tree $T$, if $G_{v}$ has solvable conjugacy problem for all $v \in V$, and if $G_{e}=\left\langle k_{e}\right\rangle$ is finite cyclic for all $e \in E$, an induction on the number of edges in $T$, using the result for the free products with amalgamation to prove the inductive step, shows that the tree product $G_{T}^{*}$ has solvable conjugacy problem. Finally, if $D$ is any nonempty finite connected graph and if $T$ is a maximal tree in $D$, an induction on the number of edges in $D-T$, using the lemma to prove the inductive step, completes the proof of the following result.

Theorem. If $D$ is a nonempty finite connected graph, if $(G, D)$ is a graph of groups, if $G_{v}$ has solvable conjugacy problem for all $v \in V$, and if $G_{e}=\left\langle k_{e}\right\rangle$ is finite cyclic for all $e \in E$, then $G^{*}$ has solvable conjugacy problem.

\section{REFERENCES}

1. K. J. Horadam, The conjugacy problem for finite graph products, Proc. Amer. Math. Soc. 106 (1989), 589-592.

2. J.-P. Serre, Trees (translated by J. Stillwell), Springer-Verlag, Berlin, 1980.

Department of Mathematics, United States Naval Academy, Annapolis, Maryland 21402 